\title{
Image classification using hybrid method
}

\author{
Ielaf O. abdl-majjed al dahl \\ Department of Computer Science \\ College of Computer Science and Mathematics \\ University of Mosul
}

Received

01 / 07 / 2010
Accepted

05 / $01 / 2011$

(المدخصن

تم في هذا البحث إيجاد طريقة تعتمد على الذمج بين الطريق تين الاعتيادية والذكائية

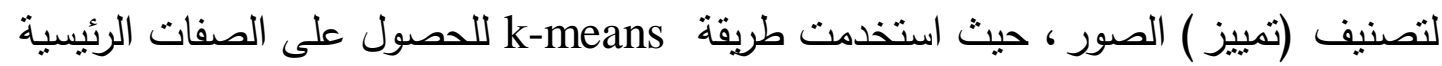

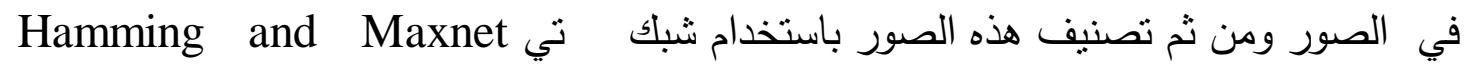
العصبية ومقارنة النتائج بالاعتماد على الطريقة الذكائية فقط ولإجراء عملية المقارنة تم استخدام ثلاثة مقياس وهي عدد خطوات التنفيذ وثانية نسبة الإثارة إلى الضوضاء (PSNR) ونالثا

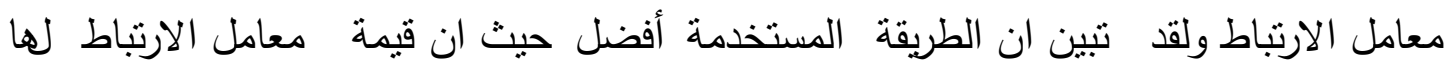

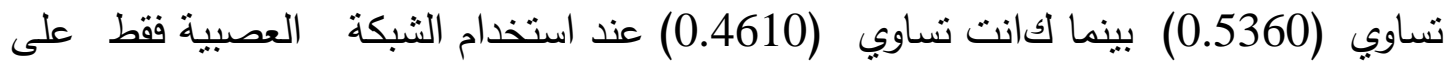
صورة من نوع JPEG. لقد طبق البرنامج على صور من نوعية JPEG و BMP واستخدم MATLAB 7.6.0 في تتفيذ برامجيات هذا البحث.

\begin{abstract}
This paper find a method depending on combining both classic and artificial methods to classify (recognition) an image.k-means method is used to find the main characteristics of the images. Then these images are classified using Hamming and Maxnet Artificial Neural Networks (ANNs). The proposed method is compared with the artificial one only. Three parameters are used for this comparison, number of iteration, second Peak Signal to Noise Ratio (PSNR) and third correlation. The results reveal that the used method is better than artificial method. correlation for proposed method is equal (0.5360), while it is (0.4610) for artificial method applying the same input JPEG image. This software is applied on JPEG and BMP gray images type. MATLAB 7.6.0 is utilized for the implementation of this software.
\end{abstract}




\section{1- Introduction}

Iteration Pattern recognition is the task performed by human being in daily life (e.g., classifying objects based on its characteristics, identifying person based on histher face etc.). All these tasks are performed by brain through learning process.

The whole idea behind pattern recognition isn't just to be able to determine if a provided pattern is exactly equal to another, but it also implies that patterns are grouped into classes. The task is then to decide which class a provided pattern belongs to [1].

The patterns belonging to a specific class share some common features. In the alphabet for example, a letter could be written in many different ways, every letter is a class embracing different patterns. No pattern recognition system can function by itself; there is always a need to some previous knowledge to base all decisions on, e.g. sample images tell what each class look like [2].

\section{2- Pattern Classification}

Pattern classification is a growing field with applications in very different areas such as speech and handwriting recognition, computer vision, image analysis, marketing, data mining, medical science, and information retrieval, to name a few. Typically, classification rules are established from randomly selected training instances from each class and are applied to test samples to evaluate their classification accuracy [3].

\section{2-1 Clustering}

The goal of clustering is to identify the clusters, which can be considered as classes [4]. Clustering is an unsupervised learning problem, which tries to group a set of points into clusters such that points in the same cluster are more similar to each other than points in different clusters, under a particular similarity metric [5].

Clustering can be used to produce an effective image index as follows: After clustering, each cluster is represented by a single representative data item (i.e. the image label for that cluster) and, instead of the original data items, the query point is compared to the cluster representatives. The best cluster or clusters, according to the used similarity measure, are then selected and the data items belonging to those clusters are retrieved also according to the used similarity measure [6].

\section{2-1-1 K-means Clustering Algorithm}

The k-means algorithm is the most frequently used clustering algorithm due to its simplicity and efficiency. K-means is a partitioned 
clustering algorithm. It performs iterative relocation to partition a dataset into $\mathrm{k}$ clusters [5]; It is based on the minimization of a performance index which is defined as the sum of the squared distances from all points in a cluster domain to the cluster center. This algorithm consists of the following steps [7]:

Step1 : Choosing $K$ initial cluster centers $z_{1}(1), z_{2}(1), \ldots, z_{K}(1)$. These are arbitrary and are usually selected as the first $K$ samples of the given sample set.

Step2 : Distributing the samples $\{x\}$ at the $k^{\text {th }}$ iterative step among the $K$ cluster domains, using the relation:

$x \in S_{j}(k)$ if $\left\|x-z_{j}(k)\right\|<\left\|x-z_{i}(k)\right\|$

for all $i=1,2, \ldots, K, i \neq j$, where $S_{j}(k)$ denotes the set of samples whose cluster is $z_{j}(k)$.

Setp3 : Computing the new cluster centers $z_{j}(k+1), j=1,2, \ldots, K$, such that the sum of the squared distances from all points in $S_{j}(k)$ to the new cluster center is minimized. In other words, the new cluster center $z_{j}(k+1)$ is computed so that the performance index $\mathbf{J}_{\mathrm{j}}$ is minimized. $J_{j}=\sum_{x \in S_{j}(k)}\left\|x-z_{j}(k+1)\right\|^{2} \quad, j=1,2, \ldots, K$

The $z_{j}(k+1)$ which minimizes this performance index is simply the sample mean of $S_{j}(k)$. Therefore, the new cluster center is given by:

$z_{j}(k+1)=\frac{1}{N_{j}} \sum_{x \in S_{j}(k)} x \quad, i=1,2, \ldots, K$

where ${ }^{N_{j}}$ is the number of samples in $S_{j}(k)$. The name "K-means" is obviously derived from the manner in which clusters are sequentially updated.

Sept4 : If $z_{j}(k+1)=z_{j}(k)$ for $j=1,2, \ldots, K$, the algorithm has converged and the procedure is terminated. Otherwise one should go to step2.

The behavior of the k-means algorithm is influenced by the number of the specified cluster centers, the choice of initial cluster centers, the order in which the samples are taken, and, of course, the geometrical properties of the data. Although no general proof of convergence exists for this algorithm, it can be expected to yield acceptable results when the data exhibit characteristic pockets which are relatively far from each other. In most practical cases the application of this algorithm will require 
experimenting with various values of $K$ as well as different choices of starting configurations [7].

\section{2-2 Neural Network Technique for Classification}

This technique has some unique advantages, such as their nonparametric nature, arbitrary decision boundary capabilities, and ability to generalize from training data. In addition, unlike traditional statistical methods, such as the maximum likelihood classifier, ANNs permit the use of a range of data types, including categorical data. It has also been reported that artificial neural networks can classify small training datasets better than conventional statistical classification technique [3].

\section{2-2-1 Hamming Neural Network as Pattern Recognizer}

Hamming Neural Network (Hamming NN) is divided into two parts one is for calculating matching score and another is MAXNET as shown in Figure(1) [8].

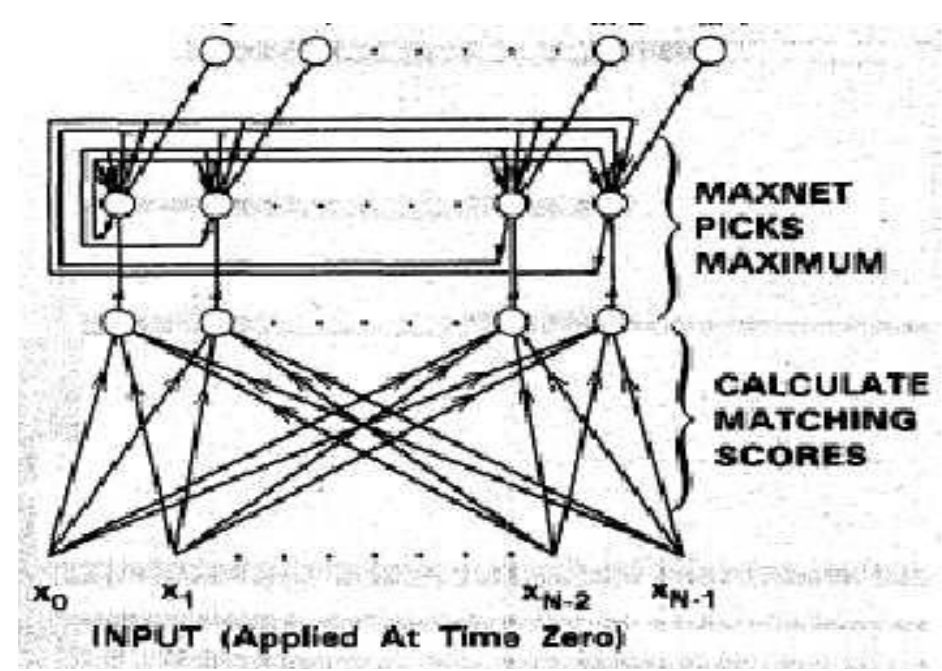

Figure 1: Hamming Neural Network

When any input is applied to this $\mathrm{NN}$, it first calculates $\mathrm{N}$ minus the Hamming distance to $\mathrm{M}$ exemplar patterns is first calculated using the former part and then the node with maximum output is found using MAXNET. The advantage of Hamming Net is that it requires fewer number of connections as compared to that of Hopfield NN [8].

The above two nets work cooperatively to identify the class to which a given input pattern belongs. The pattern is identified by means of a set of stored prototype patterns (one for each class). The input pattern is assigned to the class of the prototype that is closest in terms of Hamming distance. The basic structure is shown in Figure(2). If it is required that the classifier is executed to identify examples from different classes, both the Hamming net and Maxnet should have p outputs. The Hamming net 
gives the largest output for the prototype that is closest (has smallest Hamming distance) to the input vector. The role of Maxnet is simply to suppress all other outputs so that the right-hand vector in Figure(2) finishes with just one non-zero output, which corresponds to the pattern class identified by the Hamming net [9].

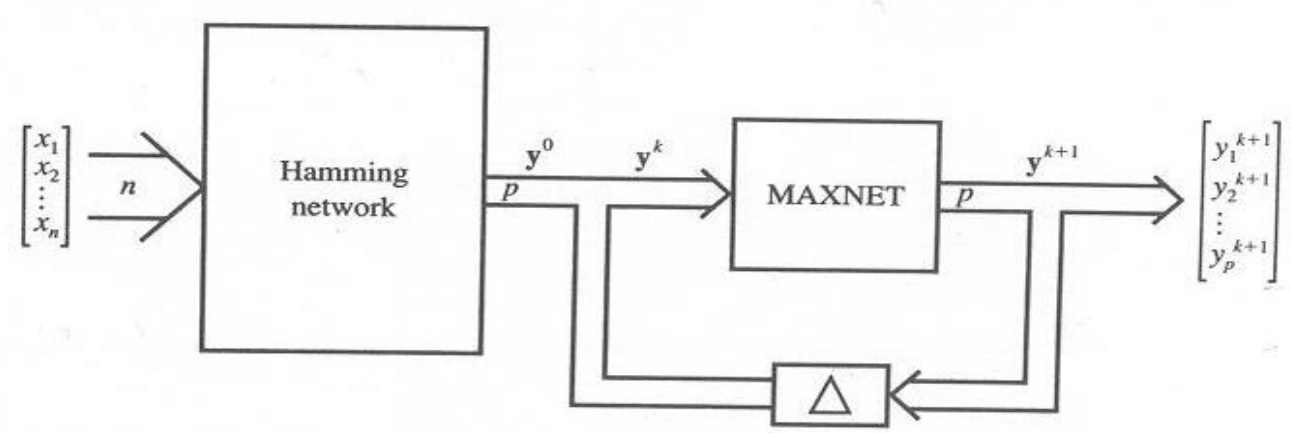

Figure2: Hamming-maxnet NN

\section{2-2-2 Hamming-maxnet algorithm [10]}

Step1 : specify the example sij (each input image convert to vector).

Step2 : fixed the weight matrix for hamming net

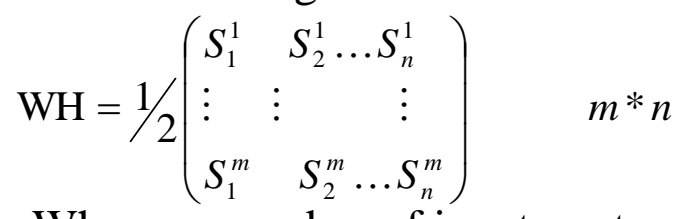

Where $n=$ number of input vector $\& m=$ number of example

Step3 : find $\theta$ where $\theta=\mathrm{n} / 2$

$$
\theta=\left[\begin{array}{l}
\theta 1 \\
\theta 2 \\
\theta 3 \\
\bullet \\
\bullet \\
\theta j
\end{array}\right] \quad \text { n=no.of vectors }
$$

Step 4 : specify the testing input vector $\mathrm{X}$, where

$$
X=\left[\begin{array}{l}
x 1 \\
x 2 \\
x 3 \\
\cdot \\
\cdot \\
x n
\end{array}\right]
$$

Step5 : find the output from hamming net

$$
\text { net } \mathrm{j}=\sum w h * x+\theta
$$

Step6 : find $y_{j k}=1 / n * n t_{j}$ 
Step7 :Find $\mathrm{Wm}$ as

$$
W m=\left[\begin{array}{lcccc}
1 & -\varepsilon & \ldots & -\varepsilon \\
-\varepsilon & 1 & \ldots & -\varepsilon \\
-\varepsilon & -\varepsilon & \ldots & 1
\end{array}\right]
$$

Where $\mathcal{E}$ is : $\theta<\varepsilon<1 / \mathrm{m}$

Step8 :find $\mathrm{yk}+1$ as

$$
\left.y_{k+1}=f \sum w m * y^{k}\right) \quad \text { where } f=\left[\begin{array}{lll}
0 & \text { if } & \text { net }>0 \\
n e t & \text { if } & \text { net }<=0
\end{array}\right]
$$

Step9 : repeat step 7 until convergence.

\section{3- Hybrid method}

In this research hybrid method used to classified image this method depend on two classical method (K-Means and Hamming and Maxnet neural network):

Step1: implementing K-Means clustering algorithm on input images.

Step2: used images from step 1 to classified by Hamming and Maxnet neural network.

\section{4- The criteria of measures}

\section{4-1- correlation coefficient}

The correlation coefficient a concept from statistics is a measure of how well trends in the predicted values follow trends in past actual values. It is a measure of how well the predicted values from a forecast model "fit" with the real-life data [11].

Correlation is a measure of the strength of relationship between random variables. The population correlation between two variables $\mathrm{X}$ and $\mathrm{Y}$ is defined as:

$\rho(\mathrm{X}, \mathrm{Y})=$ Covariance $(\mathrm{X}, \mathrm{Y}) /\{\text { Variance }(\mathrm{X}) * \text { Variance }(\mathrm{Y})\}^{1 / 2}$

$\rho$ is called the Product Moment Correlation Coefficient or simply the Correlation Coefficient. It is a number that summarizes the direction and closeness of linear relations between two variables.

The sample value (correlation) is called $\mathrm{r}$, and the population value is called $\rho$ (rho). The correlation coefficient can take values between -1 through 0 to +1 . The sign (+ or - ) of the correlation defines the direction of the relationship. When the correlation is positive $(r>0)$, it means that as the value of one variable increases, so does the other. For example, as the dose amount of an oncology medicine increases, so does the survival time, in a certain range. If a correlation is negative $(r<0)$, it indicates that when one variable increases, the other variable decreases. This means there is an inverse relationship between the two variables.

The mathematical formula for computing $\boldsymbol{r}$ is:

$$
r=\frac{n \sum x y-\left(\sum x\right)\left(\sum y\right)}{\sqrt{n\left(\sum x^{2}\right)-\left(\sum x\right)^{2} \sqrt{n\left(\sum y^{2}\right)-\left(\sum y\right)^{2}}}}
$$

where $n$ is the number of pairs of data [12]. 


\section{4-2- Peak signal-to-noise ratio (PSNR)}

The PSNR block computes the peak signal-to-noise ratio, in decibels, between two images. This ratio is often used as a quality measurement between the original and a reconstructed image. Higher PSNR indicates, better image quality.

To compute the PSNR, the block first calculates the mean-squared error using the following equation:

$$
\mathrm{MSE}=\frac{\sum_{M N}\left[I_{i}(m, n)-I_{21}(m, n)\right]^{2}}{M * N} \ldots \ldots \ldots \ldots .
$$

Where $\mathrm{M}$ and $\mathrm{N}$ are the number of rows and columns in the input images, respectively. Then the block computes the PSNR using the following equation:

$$
P S N R=10 \log _{10}\left[\frac{R^{2}}{M S E}\right]
$$

In the previous equation, $\mathrm{R}$ is the maximum fluctuation in the input image data type. For example, if the input image has a double-precision floating-point data type, then $\mathrm{R}$ is 1 . If it has an 8-bit unsigned integer data type, $\mathrm{R}$ is 255 , etc.[13].

\section{5- Results}

Experiment is performed by JPEG and BMP images type. The original, classified, and reconstructed images for the methods are shown in Figures below:

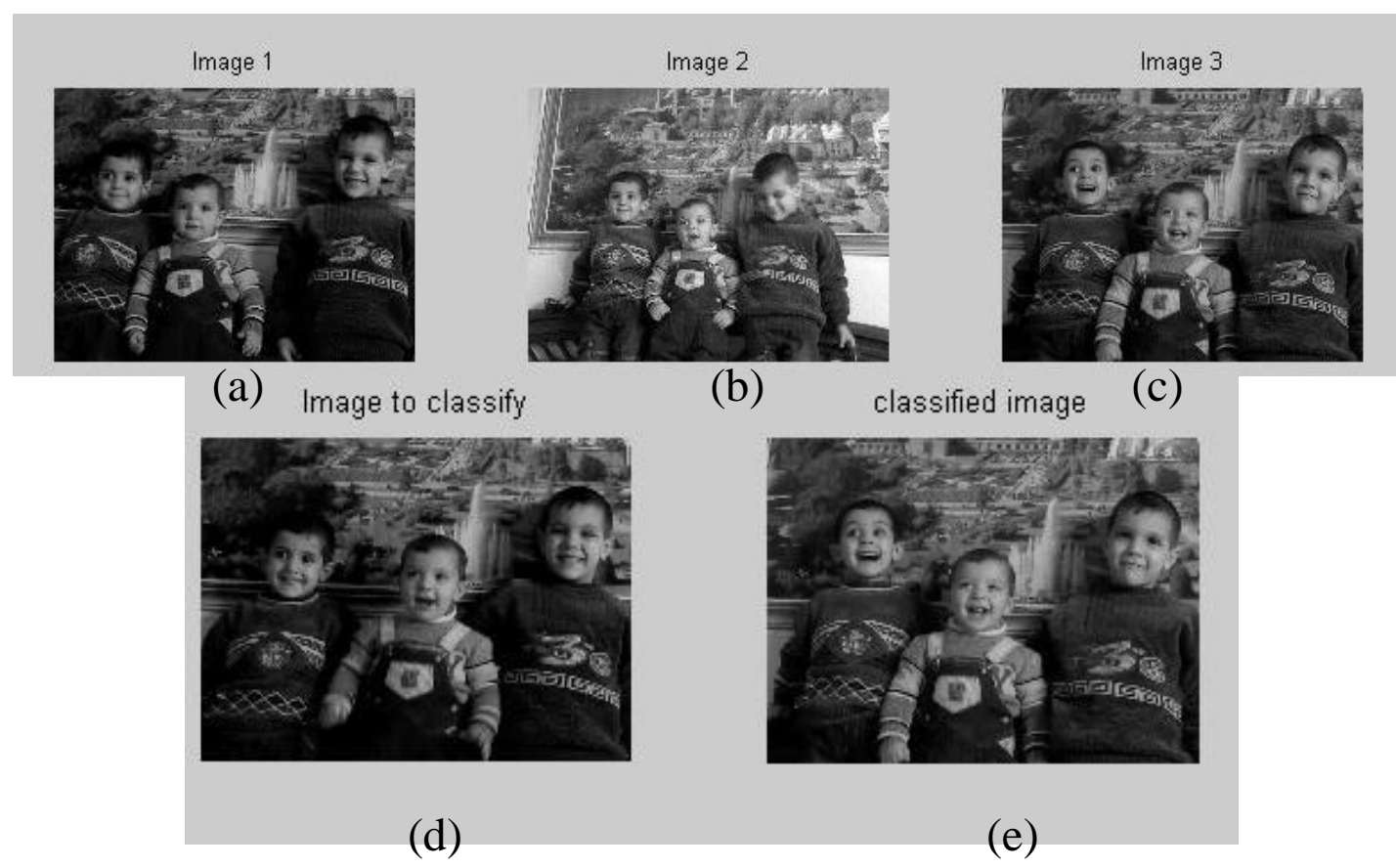

Figure 3: original, classified, and reconstructed JPEG images for Hamming and Maxnet ANNs method 
Image classification using hybrid method.

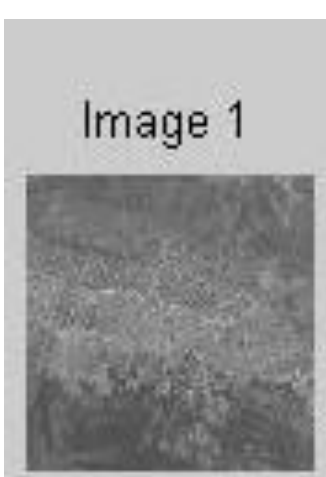

(a)
Image 2

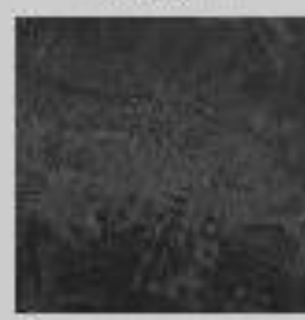

(b)
Image 3

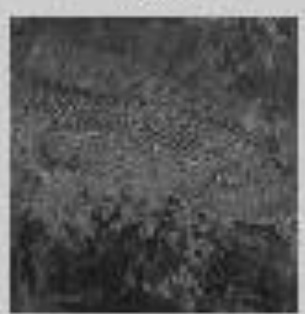

(c)

Image to classify

classified image

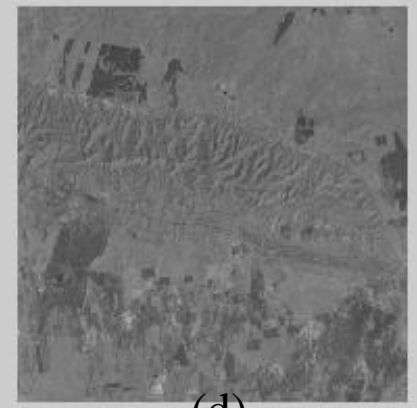

(d)

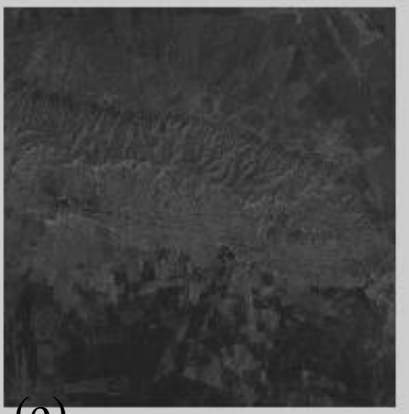

(e)

Figure 4: original, classified, and reconstruct BMP images for Hamming and Maxnet ANNs method

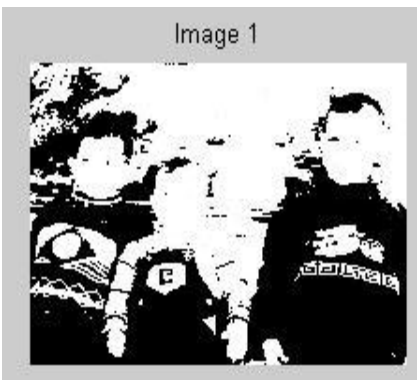

(a)

Image to classify

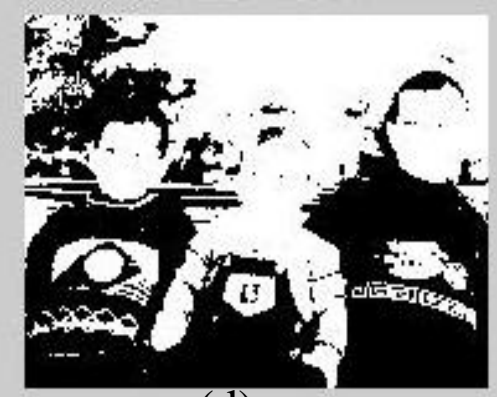

(d)

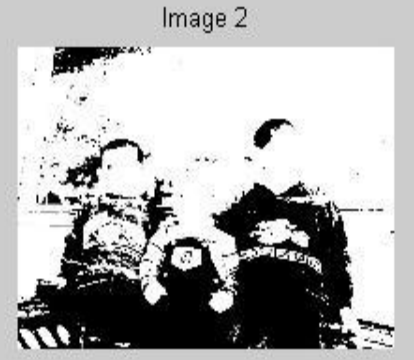

(b)

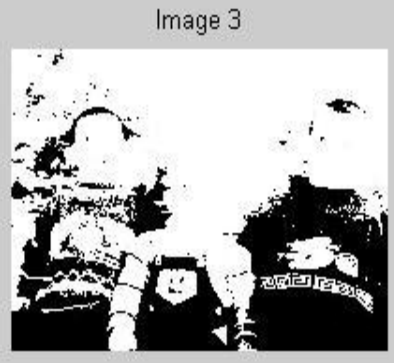

(c) classified image

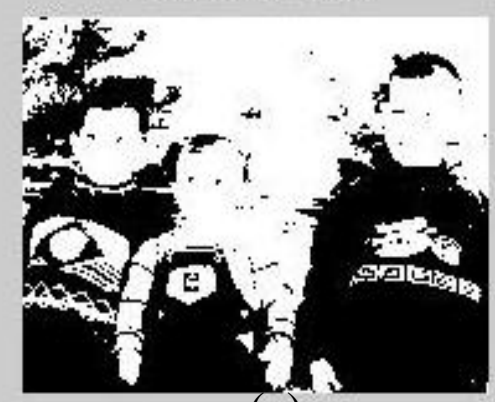

(e)

Figure 5.original, classified, and reconstructed JPEG images for the proposed method 


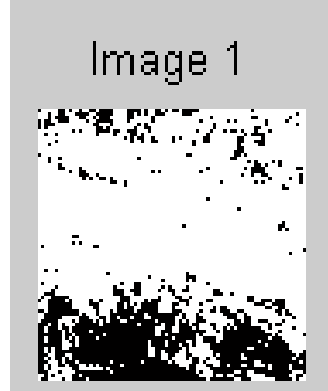

(a)

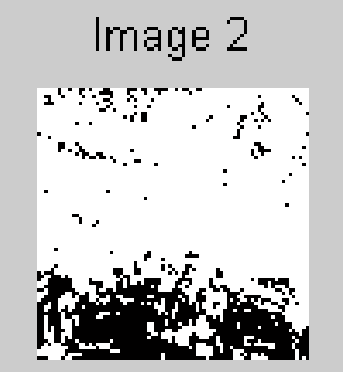

(b)
Image 3

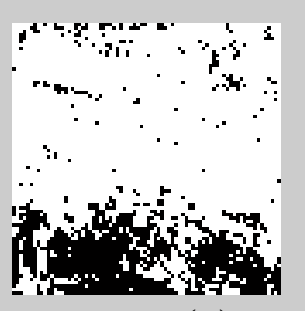

(c)

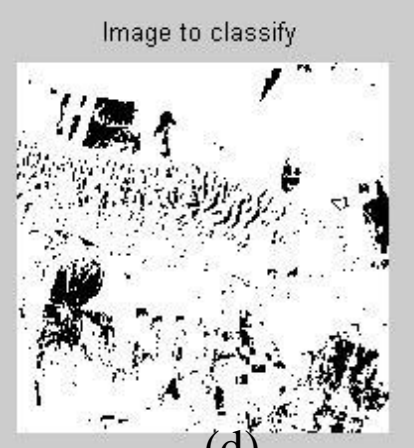

(d)

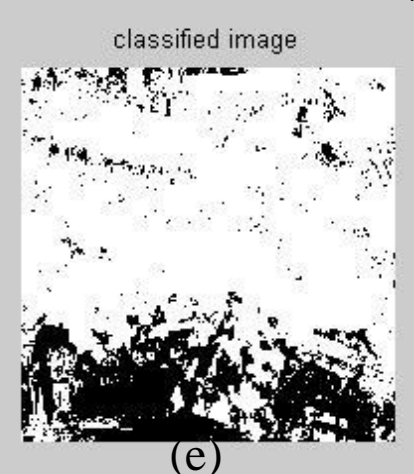

(e)

Figure 6.original, classified, and reconstructed BMP images for the proposed method

Figure (3). (a, b, and c) shows original images, (d) shows image to be classified, and (e) is reconstructed image using the images hamming and maxnet ANN on JPEG images.

Figure (4). (a, b, and c) shows original images, (d) shows image to be classified, and (e) is reconstructed image using the images hamming and maxnet ANN on BMP images.

Figure (5) (a, b, and c) shows the same original images, (d) shows image to be classified, and (e) is reconstructed image using the proposed method that applied K-means on the original and classified images then hamming and maxnet ANN used to find the reconstructed image on JPEG image.

Figure (6) (a, b, and c) shows the same original images, (d) shows image to be classified, and (e) is reconstructed image using the proposed method that applied K-means on the original and classified images then hamming and maxnet ANN used to find the reconstructed image on BMP image.

The resulting number of iteration, PSNR, and correlation values used to compare between different methods using JPEG and BMP images. These values are illustrated in table 1 and 2 .

Table1: results of measuring criteria using JPEG image

\begin{tabular}{|c|c|c|}
\cline { 2 - 3 } & Proposed method & ANN method \\
\hline Number of iteration & 10 & 11 \\
\hline PSNR & 38.9336 & 28.1073 \\
\hline correlation & 0.5360 & 0.4610 \\
\hline
\end{tabular}


Table2: results of measuring criteria using BMP image

\begin{tabular}{|c|c|c|}
\cline { 2 - 3 } & Proposed method & ANN method \\
\hline Number of iteration & 12 & 8 \\
\hline PSNR & 41.2164 & 35.2758 \\
\hline correlation & 0.1323 & 0.3369 \\
\hline
\end{tabular}

\section{6- Conclusion}

Proposed method is better because the metrics used to measure the efficiency of this method are more than the hamming method, PSNR and correlation values are greater in the proposed method than those in the ANN, and number of iterations in ANN is (11) but it is (10) in proposed method when software is applied on JPEG image, but number of iterations in ANN is (8) but it is (12) in proposed method when software is applied on BMP image.

\section{References}

1) Algorithms for pattern recognition, Joel Björnson, 2007.

2) M. Sonka, V. Hlavac, R. Boyle, _Image Processing, Analysis, and Machine Vision_, Brooks/Cole Publishing Company, International Thomson Publishing Inc., U.S.A., (1999).

3) T. Kavzoglu. An investigation of the design and use of feed-forward artificial neural network in the classification of remotely sensed images. Thesis for Ph.D., University of Nottingham, pages 11,85, May 2001.

4) Aleksandra $T$, Genetic Programming in Data Mining: Cellular Approach, MSc Thesis, Institute of Informatics Faculty of Mathematics, Physics and informatics, Comenius University, Slovakia., (2003).

5) Basu S., Semi-Supervised Clustering: Learning With Limited User Feedback, PhD Thesis, University of Texas, Austin, (2003).

6) Krishnamachari S., Abdel-Mottaleb M., Hierarchical Clustering Algorithm For Fast Image Retrieval, Conference on Storage and Retrieval For Image and Video Databases, pp. 427-435, (1999).

7) Tou J., Gonzalez R., Pattern Recognition Principles, AddisionWesley Publishing Company, USA, (1974).

8) R. P. Lippmann. An introduction to computing with neural nets. IEEE ASSP Magazine, pages 4, (22, April 1987)

9) E Oja, "Neural networks, principal components and subspaces", International Journal of Neural Systems, 1, 61-68, (1989).

10) Jaeek m. zurada: Introduction to artificial neural network system (1998).

11) (C) 1997-2005. Financial Forecast Center LLC. All Rights Reserved. " http://forecasts.org/cc.htm",[internet].

12) Zaizai Lu, Computation of Correlation Coefficient and Its Confidence Interval in SAS®, David Shen, WCI, Inc, (2000).

13) MATLAB 7.6.0(R2008), 1984-2008 The Math Works, Inc. 